\title{
Use of modern technology as an aid to medication adherence: an overview
}

This article was published in the following Dove Press journal:

Patient Intelligence

I June 201 I

Number of times this article has been viewed

\author{
Sandra C Thompson \\ Adrian T Walker \\ Combined University Centre for \\ Rural Health, University of Western \\ Australia, Western Australia, \\ Australia
}

\begin{abstract}
As life expectancy increases, and with it the increased burden of chronic disease, there is increasing reliance on pharmaceuticals for primary and secondary prevention. However, adherence to the regime of treatment prescribed by the doctor is suboptimal for a wide range of reasons. This article explores the role of modern technology in trying to improve adherence to prescribed medications by searching peer-reviewed articles through the online PubMed and Google Scholar databases using the keywords "adherence", "compliance", "medication", "pharmaceutical", and "technology", and further citation snowballing. Literature was reviewed to determine reasons for noncompliance and how technology is being used to improve patient adherence. The following factors potentially assist patients to maintain good adherence by helping minimize side effects or simplifying doses and regimens: electronic prescribing and use of shared electronic health records so that all care providers are aware of prescribed medications; reduced costs through improved manufacturing processes; dosing and appointment reminders and dosing aids; improved drugs which are more acceptable to the patient and have reduced side effects; and better condition monitoring by doctors and patients. The areas which are least amenable to positive changes are those that are intrinsic to the patient, such as self-efficacy and motivation, and the quality of the doctor-patient interaction, which impacts upon patients' understanding of their disease and their trust and confidence in their health care provider. While many new approaches to assist adherence are reported regularly in the media and on the Internet, there is frequently little high-quality evidence to confirm the utility of the approach. Improving adherence is likely to require interventions at many levels. The doctor-patient relationship and interventions supporting patients' understanding of their disease and confidence in the therapy prescribed remain important areas for further research.
\end{abstract}

Keywords: compliance, adherence, medication, pharmaceutical, technology

\section{Introduction}

Medicine uses modern technology to help in man's efforts to sustain and prolong life and improve quality of life. Whether it is through the invention of new modalities of imaging or mechanical tools to aid in surgical techniques, technology has continued to allow societies to progressively raise outcomes as a result of health care. Pharmaceuticals are an important component of modern medical treatments. As a major intervention and treatment modality, dosage and timing recommendations are based upon pharmacodynamics and clinical trials to determine schedules that elicit the most useful therapeutic action with the fewest and least severe side effects. ${ }^{1}$ Over US\$450 per person per year is spent on prescription medication in Australia, and around double that (US\$897/person per year) in the US, with the number and cost of pharmaceuticals continuing to grow. ${ }^{2}$ There is little
Correspondence: Sandra CThompson Combined University Centre for Rural Health, University of Western Australia, 167 Fitzgerald St, Geraldton 6530, Western Australia, Australia

$\mathrm{Tel}+61899560208$

Fax +6189964 2096

Email sandra.thompson@cucrh.uwa.edu.au 
point in doctors prescribing medications, and governments and consumers paying for pharmaceuticals, unless they are used in a way that is therapeutic, as demonstrated in clinical trials that show efficacy. Therefore, it is important that medications are used by ensuring patients adhere to doses and schedules for which there is evidence of benefit. As such, patient adherence continues to be paramount in the efficacy of drug treatment. Given that compliance is a term used in the medical literature and considered to have judgmental overtones, the terms adherence or concordance (which implies an equal partnership between the patient and prescriber) are preferred. However, particularly in older reviews, the term compliance is still widely used. ${ }^{3}$ For the purposes of this review, we have used the term adherence. Many factors play a part in determining the adherence with a particular drug, and the World Health Organization has categorized the factors influencing adherence in the long-term into five areas, ie, social and economic, health care team and system, therapy-related, condition-related, and patient-related factors. $^{4}$

Modern technology can help to increase adherence through ways in which drugs can be packaged, dosing schedules and delivery, reducing prescription errors, monitoring of drug use, and reducing adverse physiological reactions. This review identifies ways in which patient adherence with medications has been assisted via the use of modern technology.

\section{Methods}

We undertook a search of peer-reviewed articles through the online PubMed and Google Scholar databases using the keywords "adherence", "compliance", "medication”, "pharmaceutical", and "technology". In addition, Google was used with the same search terms to identify information in the grey literature. "Decision support" was not included as a search term, although decision support tools may influence doctors' prescribing and hence, patient adherence. Similarly "patient decision aids", ie, tools to involve patients in making choices about their treatment, were not included in our search strategy, which focused more on supporting patients after medications had been prescribed. After the initial search, further articles were identified by citation snowballing and searching for specific technologies to identify relevant articles. The aim was to distill information to identify factors important in adhering to prescribed medications and how technology is being used in ways to improve patient adherence.

\section{What is adherence?}

Adherence refers to the extent to which the patient's actual history of drug administration corresponds to the prescribed regimen, ie, taking the correct dose of the correct medication at the correct time. ${ }^{3}$ There are a number of forms that poor adherence may take, including receiving a prescription but not having it filled at the pharmacy, taking the incorrect dose, forgetting doses of medication (or increasing the frequency), and stopping the treatment too soon (either by ceasing it too soon or failing to obtain a refill).

Failure to fill a prescription is impacted upon by the cost of the drug and the cost of any copayment, with more expensive drugs more likely not to be filled. ${ }^{5}$ Two main categories of dosing are defined, ie, dose-taking (taking the correct dose each day) and dose-timing (taking pills within the correct time period). Various approaches have been used to deal with both of these failures to take medicine as prescribed, but studies continue to show that a large proportion of patients fail to comply with prescribed medications in one way or another.

\section{How to measure adherence}

Traditionally, indirect measures, including interviews, diaries, and tablet counts, were used to assess patient adherence. More recently, modern technology, such as microprocessors in the caps of pill bottles, have been used to assess whether medication is accessed and when the pill bottle is opened, but even this is not an indication of whether the drug dose is actually taken. More direct measures, such as measuring pharmacologic markers and drug levels in urine or blood, can also be used to determine whether an effective dose of a drug is achieved. Recently, at least in the context of drug trials, labeling of drugs with a slow turnover marker and a long half-life, such as low-dose phenobarbital, enables accurate ascertainment of the drug dose taken during the prior 10 days. ${ }^{6}$ This overcomes one of the problems with measuring drug levels in urine or blood, ie, that if the drug is taken more regularly over the last few days before evaluation than during the rest of the time since prescribing (the "toothbrush" effect), the phenobarbital level will reflect this and more accurately reflect true adherence. ${ }^{7}$

\section{Extent and consequences of drug nonadherence}

Substantial literature exists on the problems of poor drug adherence. The advent of electronic monitoring has made even more evident the extent to which patients fail to adhere with physicians' instructions and labeled drug use. ${ }^{6}$ Over one-third of patients are poorly compliant with prescribed drug regimes, apparently with no statistically significant difference in regard to disease or prognosis. ${ }^{8}$ Nonadherence with medications is a huge problem in health care provision, associated with increased costs 
arising from additional admissions to hospital, longer length of stay, and higher hospital expenditure. A substantial proportion of admissions to nursing homes are also attributed to patients' inability to manage their therapy adequately. ${ }^{4,9}$

\section{Causes of nonadherence}

There are many reasons why patients may not be adherent with prescribed medications. One issue has been the number of drugs that patients are often required to take given the increasing prevalence of chronic diseases, and different requirements for dosing time and instructions around taking medications with or without food. This complexity of the regime has been considered by some as the responsibility of the doctor who prescribes multiple medications for a patient. ${ }^{9}$ However, the increasing age of the population and the increase in chronic conditions that have been shown to extend life and improve quality of life when managed, accounts for much of the complexity of drug regimens. However, adherence is likely to be particularly affected when patients are unable to follow multiple or complex instructions, such as those who are elderly or have memory problems.

Patients' inability to understand or remember instructions and the reasons for the medication account for at least some of the problems of nonadherence. This can be considered as a problem of communication between the doctor and patient. Patients are often anxious when seeing the doctor, and this increases their chance of not being able to recall what was said to them in the consultation. Apart from doctors using technical language which is not well understood by the average patient, time pressures and interruptions may mean that the doctor fails to provide an explanation that is understood by a layman, apart from doctors not really understanding patients' beliefs about the diagnosis and disease and what it is that they don't know. It is also well-known that patients' anxiety when they see the doctor means they may fail to remember what was said in the consultation. ${ }^{10}$ Evidence shows that the involvement of a pharmacist can help to improve adherence, particularly with asymptomatic conditions such as hypertension, although the best way for this intervention to occur requires further exploration. ${ }^{11}$

The cost of medications may also be a reason why some patients are not compliant with taking medications prescribed by their doctor. If a person cannot afford the treatment, they may well be ashamed to admit this to their doctor, and it means that they may not fill the prescription for the treatment of an acute condition, let alone do it on a recurring basis to prevent the long-term adverse consequences from a chronic disease that is not currently causing any symptoms and where the medication itself may have adverse effects. Reduced costs of medications can lead to an increase in adherence, and there is little evidence for the economic theory that suggests patients who pay for their medicine are more likely to attach value to it, and therefore are more likely to adhere to their regimen. ${ }^{12}$ However, it is clear that free medicine does not ensure perfect adherence, with one study showing rates of $40 \%$ nonadherence even when no copayment was required. ${ }^{13}$

Finally, patients have unresolved concerns around the diagnosis, lack of symptoms, time between taking a medication and its effect, and fear or misunderstanding of adverse effects. ${ }^{14}$ Donovan argues that patients make decisions based upon their own beliefs and the information available to them, attempting to reach a level of medication that suits their own particular circumstances. Patients with chronic illnesses make decisions about treatments that fit into their own beliefs and personal circumstances.

Multiple factors may contribute to a patient being noncompliant. Each of the major reasons contributing to nonadherence represents only one component of a complex picture, and for each component there are different modern technologies that could potentially contribute to better adherence, as summarized in Table 1. However, it is important to recognize that multiple factors contribute to a patient receiving and taking the right dose of a drug in the right way and at the right time.

\section{Tackling nonadherence using modern technology}

Modern technology has contributed in multiple ways to us having a better understanding of the biological and chemical mechanisms underpinning health and illness, opening up the possibility of treatments, many of which are pharmacological. Understanding underlying mechanisms and the creation of better treatments does not, however, translate into benefit until they are utilized in a way where there is evidence of benefit. However, an overwhelming number of studies have shown that adherence has a positive impact on health service utilization, and on clinical and economic outcomes.

The development of computerized systems for prescribing, linked to an online pharmacopeia which includes help with identifying indications, contraindications, side effects and drug interactions, can also improve the legibility and accuracy of prescriptions and often the timeliness with which they are available to the pharmacist. Such developments can be described as offering safer, more efficient, and effective prescribing practice and can help with the patient actually having the medication which the doctors intends them to take. 
Table I Summary of contributors to poor adherence and potential technological solutions

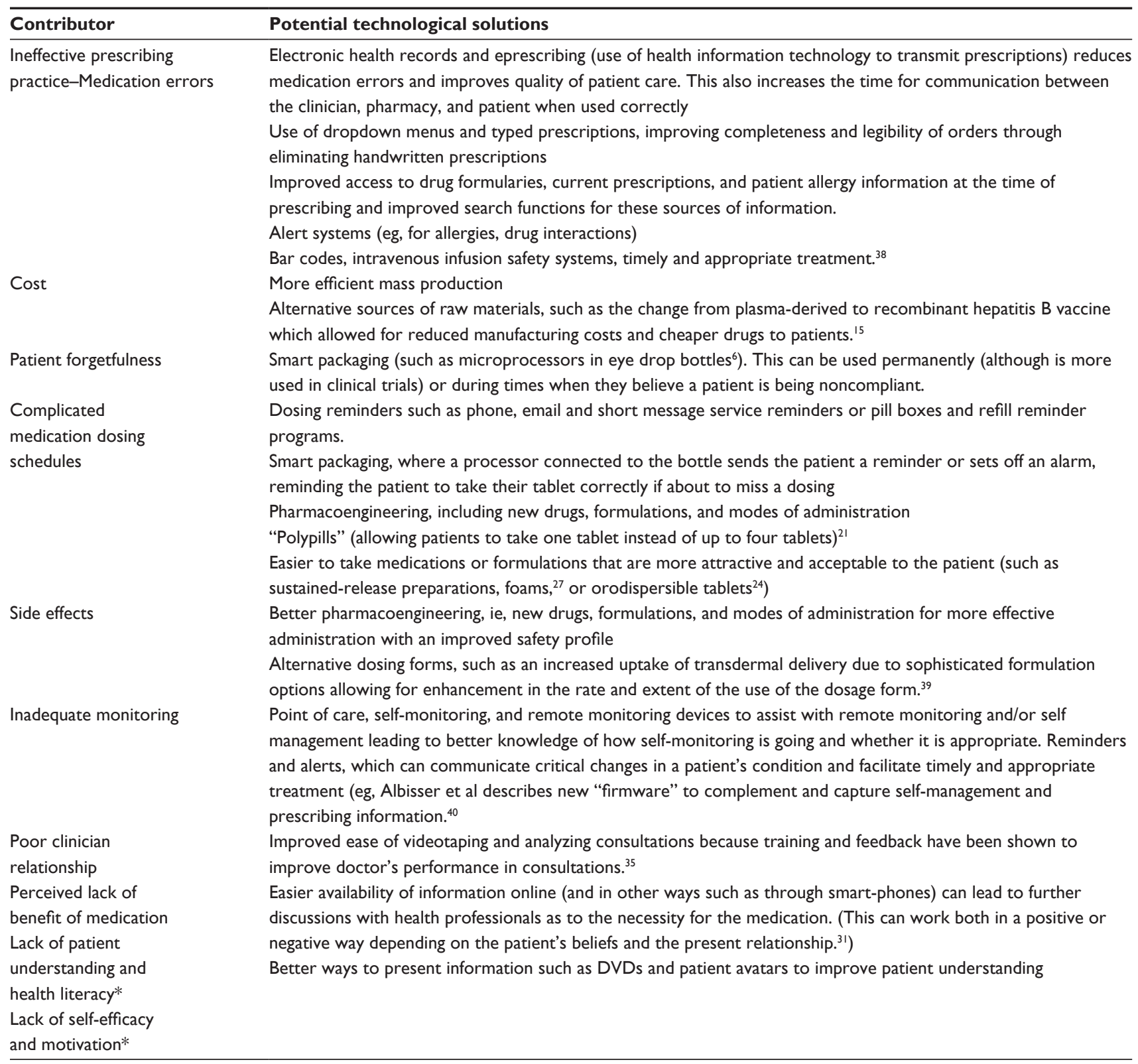

Note: *These contributors to poor adherence are the least amenable to modern technological interventions.

Cost is a major factor in whether patients actually fill or refill their prescription, so things which help lower the cost of medications to the consumer may help improve adherence. For some therapeutics, modern technology has helped lower the cost of a drug, and often improved the safety of a product. An example of this is the change from plasma-derived to recombinant hepatitis B vaccine or human growth hormone. ${ }^{15}$ Given the level of regulation of prescription medications in developed countries, the move to encourage generic prescribing is an important means of trying to contain costs. However, the Internet now offers a low-cost online source of brand name and generic drugs, although it is doubtful whether access in this way actually increases adherence with doctor-prescribed medications. Patients may seek more information and interaction, often via the Internet, to understand and manage their condition, which can include advice on how to reduce the costs of expensive medications, such as drug treatments for cancer. Suggestions include that patients consult their doctor about the possibility of prescribing alternative medications that are less expensive. Consumers are often encouraged to ask their pharmacist about are the possibilities of finding a generic alternative to a prescribed cancer medication, or other effective generics which may be off patent and therefore able 
to be legally copied by competing companies. These are usually competitively priced because of competition between different drug manufacturers, and can be potentially much cheaper than the same "brand name" medication. ${ }^{16}$ However, whilst addressing financial barriers is essential to improve adherence, it is only one important step.

Various aids to dosing are now regularly used to assist in patients complying with medication. Given that many patients with chronic diseases often have a number of different medications to be taken with different schedules, including an increasing number of elderly patients who have cognitive and memory problems, many pharmacies and carers have increased the packaging of medications in dosette boxes or the more foolproof Webster packs. While not a high-technology device, Webster packs do use modern technology to seal the medication securely into a blister pack to protect medications from being accidentally mixed up, and which makes clear the day and time when the dose should be taken. The pack is transparent on one side, so it is easy to see what medication has been removed through the foil backing. However, while blister packs appear to lead to more pills being taken, the evidence for better adherence is not strong. ${ }^{17}$

Technology has delivered even more sophisticated pill box systems, such as one commercial product which links via wireless to a central computer and monitors the patient's dosage activity. ${ }^{18}$ As described, this can be viewed online by carers and is programmed remotely via the Internet or through the company over the phone; once set up, it provides patient reminders complete with flashing lights, beeps, automatic phone calls, text messages, and emails, depending on how the system is programmed. The patient's activity log can be viewed online by caregivers and healthcare providers, and the system can be programmed to send real-time email or text message notifications or weekly email reports. If the wrong medication cup is taken, the system sounds an alert, ensuring that patients take the correct medication. While targeted at elderly people living alone and marketed at those concerned for them, rigorous evidence for better outcomes by using such a system is not yet available.

The increasing ownership of mobile phones, or similar personal items carried close to owners more or less constantly, combined with the low-cost nature of short message services and ability to automate message sending, has led to a large number of trials of use of phone calls and short message services for health-related calls. This has been used particularly for appointment reminders and by supporting reminders of medication doses, therefore improving adherence. It is used in developing countries to deliver health care at low cost in circumstances where medication dosing is particularly important, and many studies have now showed the benefits in the case of antiretroviral medications for human immunodeficiency virus. ${ }^{19}$ Apps for Smart phones can also provide reminders to be set and may suit the technologically savvy, as well as enabling other information such as side effects to be recorded.

The formulation of new or improved medications and drugs is one way in which technology offers hope for improving adherence. Numerous examples exist of new drugs, developed through scientific study and experiments, which have improved upon previously available treatments. This may be through pharmacoengineering to reduce the frequency of dosing, and a reduction in side effects. The annoyance of remembering to take medications several times a day has been one reason patients give for omitting doses. The desire to simplify drugtaking regimes has led to efforts to reduce the frequency of dosing, given that some evidence has suggested that adherence is inversely associated with dosing frequency, and more convincing evidence shows that simpler, less frequent dosing improves adherence across a variety of therapeutic classes. ${ }^{20}$ Long-acting preparations and pill combinations require careful science and engineering to ensure medications remain safe and effective without increasing the number of side effects or drug interactions. Careful formulation is also required to combine several medications together to create "polypills" for conditions such as ischemic heart disease. These strategies aim to facilitate adherence by simplifying a complex regimen (such as one pill instead of four). Although a promising area in which many trials are occurring, arguably even the use of polypills will still need to be combined with other methods for improving adherence to utilize them for their best effect. ${ }^{21-23}$ New technologies have also enabled the production of new formulations, such as orodispersible tablets for those who have difficulty swallowing pills ${ }^{24}$ or capsules to encase the taste of unpalatable medications. One example is through the development of nanotechnology which manipulates matter at the atomic or molecular level and has made possible the production of nanocarriers and microcarriers to target bioactive compounds in lung disease more effectively. ${ }^{25}$ Through a variety of particles never dreamed of some years ago, ie, nanocarriers and microcarriers, such as microspheres, nanoparticles, liposomes, niosomes, and dendrimers, the targeted delivery of bioactive molecules to the lungs can occur. Such carriers can aid sustained drug delivery to the lungs, extend the duration of action, reduce the therapeutic dose, improve patient adherence, and reduce adverse effects from highly toxic drugs. Improvements in antiretroviral treatments for human immunodeficiency virus over the last few years highlight the 
role that technology has played in drug development. ${ }^{26}$ Another example is the use of foam vehicles for topical products which have good safety profiles and their increasing use in the community. Zhao et al state that there is no clinical evidence for foams being superior to the more traditional vehicles, such as creams or ointments. However, their effectiveness may be increased because consumers find foams more "elegant, aesthetic, and cosmetically appealing", so they are more likely to use them, and more likely to use them appropriately. ${ }^{27}$

The rationale for prescribing drugs is to intervene in a disease process and/or to improve symptoms. Our understanding of the natural history and the pathological processes of disease continues to improve. Given the increasing burden of chronic diseases on the health system, there is strong support for delivering evidence-based drug treatments for primary or secondary prevention, at a time when the conditions are still asymptomatic, to minimize disease processes which cause harm in the long-term. In these circumstances, point of care testing devices which provide feedback on the control of a disease may help improve patient adherence. Excellent evidence exists for this in relation to glycemic control, although less evidence exists for benefit in hypertension and international normalized ratio testing for coagulation control. ${ }^{28}$ Other remote monitoring devices have not enjoyed the same benefits for patients or uptake, although further refinement of devices and reductions in cost may help. An example of this is remote blood pressure monitoring, where the cost and size of devices has been an impediment to use and uptake.

A patient's interaction with their doctor (and the health care system) sets the scene for whether a patient is likely to have confidence in the advice received and to take prescribed medications. The importance of trust and confidence in the physician, of being listened to and having their concerns addressed, ${ }^{29}$ patients' beliefs, ${ }^{30}$ and confidence in the diagnosis and understanding of the rationale for a drug being taken is critical in patient adherence. Technology can have a positive or a negative impact on these areas, depending on the attitude of the physician. The availability of information online (and a lack of understanding on the part of both physicians and patients as to its verifiability) can lead to a lack of desire to take new medicines if the risks behind them are not put into context. This can be time-consuming for the physician, although the time spent addressing concerns can strengthen the relationship between physician and patient, occasionally leading to changes in the diagnosis, and therefore indirectly improving adherence. ${ }^{31}$ While new technologies can help with health care provider information through opportunities for distance education and professional development, it has been recognized that generally there is too little attention to training specifically about adherence. ${ }^{4}$ Even in an era where there is considerable focus on self-management, the issues raised by Donovan remain of critical importance. ${ }^{9,14,32}$ Donovan argued that the patient's role in medical decisionmaking was largely absent in compliance research and that patients make their own reasoned decisions about treatments, based upon their own beliefs, circumstances, and the information available to them. Lack of information and skills in selfmanagement, difficulty with motivation and self-efficacy, and lack of support for behavioral change are all impediments to patient adherence. ${ }^{4}$ The push for self-management or involving the patient in the management of their own chronic disease is a relatively recent development, and can still be met with some resistance, depending on the condition being treated. However, there is good evidence for this strategy in combination with other means to improve adherence, particularly because it allows modifications based on the patient's lifestyle. ${ }^{33}$ Education on its own is a weak intervention, but information and understanding are nevertheless important components that contribute to patients being motivated to take a health care provider's advice. Advances in ways of presenting information is a new frontier to be explored in terms of improving adherence, ${ }^{34,35}$ and may contribute to effective multicomponent interventions.

Some modern technologies, despite good intentions, have made adherence more difficult. A good example of this is childproof caps, because even though efforts have been made to make them easy for adults to open, some patients, particularly the elderly, still find them difficult. Some patients discontinue the use of medicines in such containers. ${ }^{36}$ Thus, while childproof containers play an important safety role, there remains room for improvement in this area.

\section{Conclusion}

There have been many ways in which the ingenuity of man and his capacity for problem solving and invention have contributed to medical advances that assist with the hugely improved life expectancy humans are enjoying in the modern world. Although medications to help control diseases are improving and becoming increasingly sophisticated in their development, adherence remains a major concern. New drugs, formulations, and approaches to improve adherence to treatment are constantly being developed and trialed. Use of multiple approaches to assist adherence are more effective than any one measure alone. ${ }^{37}$ Despite all that modern technology offers, patient beliefs, self-efficacy and motivation, and the doctor-patient relationship remain hugely important, reflecting the old adage that the practice of medicine is an art not just a science. 


\section{Disclosure}

The authors report no conflicts of interest in this work.

\section{References}

1. Urquhart J. The drugs not taken. Science. 2008;321:769.

2. Organisation for Economic Co-operation and Development. OECD Health Data: Selected Data. OECD Health Statistics. 2010. Available at: http://www.oecd-ilibrary.org/content/data/data-00348-en. Accessed May 9, 2011.

3. Fraser S. Concordance, compliance, preference or adherence. Patient Prefer Adherence. 2010;4:95-96.

4. World Health Organization. Adherence to long-term therapies: Evidence for action. Geneva, Switzerland: World Health Organization; 2003.

5. Shrank WH, Choudhry NK, Fischer MA, et al. The epidemiology of prescriptions abandoned at the pharmacy. Ann Intern Med. 2010;153: 633-640.

6. Urquhart J, De Klerk E. Contending paradigms for the interpretation of data on patient compliance with therapeutic drug regimens. Stat Med. 1998; 17:251-267.

7. Hardy E, Kumar S, Peaker S, Feely M, Pullar T. A comparison of a short half-life marker (low-dose isoniazid), a long half-life pharmacological indicator (low-dose phenobarbitone) and measurements of a controlled release 'therapeutic drug' (metoprolol, Metoros) in reflecting incomplete compliance by volunteers. Br J Clin Pharmacol. 1990;30:437-441.

8. Urquhart J. Patient non-compliance with drug regimens: measurement, clinical correlates, economic impact. Eur Heart J. 1996;17 Suppl A: $8-15$.

9. Donovan JL. Patient decision making. The missing ingredient in compliance research. Int J Technol Assess Health Care. 1995;11:443-455.

10. Ley P. Memory for medical information. Br J Soc Clin Psychol. 1979;18: 245-255.

11. Roughead L, Semple S, Vitry A. The value of pharmacist professional services in the community setting: A systematic review of the literature 1990-2002. Adelaide, Australia: University of South Australia; 2002.

12. Dor A, Lage MJ, Tarrants ML, Castelli-Haley J. Cost sharing, benefit design, and adherence: the case of multiple sclerosis. Adv Health Econ Health Serv Res. 2010;22:175-193.

13. Elliott R. Non-adherence to medicines: not solved but solvable. J Health Serv Res Policy. 2009;14:58-61.

14. Donovan JL, Blake DR. Patient non-compliance: Deviance or reasoned decision-making? Soc Sci Med. 1992;34:507-513.

15. Stephenne J. Recombinant versus plasma-derived hepatitis B vaccines: issues of safety, immunogenicity and cost-effectiveness. Vaccine. 1988;6:299-303.

16. Jordan T. How to lower the cost of cancer drugs. 25 January 2011. Available at: http://www.ehow.com/how_5733182_lower-cost-cancerdrugs.html\#ixzz1BxYSAau. Accessed May 9, 2011.

17. Huang H, Maguire M, Miller ER, Appel L. Impact of pill organizers and blister packs on adherence to pill taking in two vitamin supplementation trials. Am J Epidemiol. 2000;152:780-787.

18. MedMinder. MedMinder Pill Dispenser System. 25 January 2011. Available at: https://www.medminder.com/Index.aspx. Accessed May 9, 2011.

19. Mukund Bahadur KC, Murray PJ. Cell phone short messaging service (SMS) for HIV/AIDS in South Africa: a literature review. Stud Health Technol Inform. 2010;160(Pt 1):530-534.

Patient Intelligence

\section{Publish your work in this journal}

Patient Intelligence is an international, peer-reviewed, open access journal that characterizes and measures the central role of patient behavior and intention in optimizing healthcare management in all areas of disease and complaint types. An improved understanding of patient intelligence coupled with predictive analysis helps an organization contribute more effectively to achieving better outcomes. Submit your manuscript here: http://www.dovepress.com/patient-intelligence-journal
20. Claxton AJ, Cramer J, Pierce C. A systematic review of the associations between dose regimens and medication compliance. Clin Ther. 2001;23: $1296-1310$

21. Elley CR, Toop L. A polypill is the solution to the pharmacological management of cardiovascular risk. J Prim Health Care. 2009;1:232-236.

22. Soliman EZ, Mendis S, Dissanayake WP, et al. A polypill for primary prevention of cardiovascular disease: A feasibility study of the World Health Organization. Trials. 2011;12:3.

23. Olsen P. "Polypill": Pros and cons.18 April 2011. Available at: http:// www.sld.cu/galerias/pdf/servicios/hta/polypill_pros_and_cons.pdf. Accessed May 9, 2011.

24. Navarro V. Improving medication compliance in patients with depression: use of orodispersible tablets. AdvTher. 2010;27:785-795.

25. Kurmi BD, Kayat J, Gajbhiye V, Tekade RK, Jain NK. Micro- and nanocarrier-mediated lung targeting. Expert Opin Drug Deliv. 2010;7: 781-794.

26. Boone LR, Koszalka GW. Antiretroviral drug development for HIV: Challenges for the future. Curr Opin Investig Drugs. 2010;11:863-867.

27. Zhao Y, Jones SA, Brown MB. Dynamic foams in topical drug delivery. J Pharm Pharmacol. 2010;62:678-684.

28. [No author listed]. INR self-monitoring and oral anticoagulants. Prescrire Int. 2010;19:130-132.

29. Meyer S, Ward P. Do your patients trust you? A sociological understanding of the implications of patient mistrust in healthcare professionals. Australasian Medical Journal. 2008:1.

30. Chia LR, Schlenk EA, Dunbar-Jacob J. Effect of personal and cultural beliefs on medication adherence in the elderly. Drugs Aging. 2006;23: 191-202.

31. Ahmad F, Hudak PL, Bercovitz K, Hollenberg E, Levinson W. Are physicians ready for patients with Internet-based health information? J Med Internet Res. 2006;8:e22.

32. Donovan JL, Blake DR, Fleming WG. The patient is not a blank sheet: Lay beliefs and their relevance to patient education. Br J Rheumatol. 1989;28:58-61.

33. Aronson JK. Compliance, concordance, adherence. Br JClinPharmacol. 2007; $63: 383-384$

34. Forbes D, Sidhu A, Singh J. Coupling of indigenous-patient-friendly cultural communications with clinical care guidelines for type 2 diabetes mellitus. Health Informatics and Knowledge Management Conference; 2011 Jan 17; Perth, Australia.

35. Bylund CL, Brown R, Gueguen JA, Diamond C, Bianculli J, Kissane DW. The implementation and assessment of a comprehensive communication skills training curriculum for oncologists. Psychooncology. 2010;19:583-593.

36. Cassel C, Leipzig R, Cohen H, Larson E, Meier D. Geriatric Medicine: An Evidence-based Approach. 4th ed. New York: Springer-Verlag; 2003.

37. Haynes RB, Ackloo E, Sahota N, McDonald HP, Yao X. Interventions for enhancing medication adherence. Cochrane Database Syst Rev. 2008;2:CD000011.

38. Forni A, Chu HT, Fanikos J. Technology utilization to prevent medication errors. Curr Drug Saf. 2010;5:13-18.

39. Mitra A, Wu Y. Topical delivery for the treatment of psoriasis. Expert Opin Drug Deliv. 2010;7:977-992.

40. Albisser A, Alejandro R, Sperlich M, Ricordi C. Closing the circle of care with new firmware for diabetes: MyDiaBase+RxChecker. J Diabetes Sci Technol. 2009;3:619-623.

The journal is characterized by the rapid reporting of reviews, original research, methodologies, analytics, modeling, clinical studies and patient surveys across all disease areas. The manuscript management system is completely online and includes a very quick and fair peer-review system. Visit http://www.dovepress.com/ testimonials.php to read real quotes from published authors. 